OPEN ACCESS

Edited by:

Demin Cai,

Yangzhou University, China

Reviewed by:

Rouzha Zlatanova Pancheva, Medical University of Varna, Bulgaria

Alyssa Susanto,

The University of Sydney, Australia

${ }^{*}$ Correspondence:

Yazhuo Liu

Yazhuo_liu@126.com

Simiao Tian

Tiansimiao@dlu.edu.cn

Specialty section

This article was submitted to

Nutrition and Metabolism,

a section of the journal

Frontiers in Nutrition

Received: 12 July 2021 Accepted: 27 September 2021

Published: 22 October 2021

Citation:

Li Y, Shen H, Li Y, Bi M, Bi Y, Che X, Tian S and Liu Y (2021) Sex-Specific

Differences in the Associations Between Omega-6 Polyunsaturated Fatty Acids and Type 2 Diabetes in

Chinese People.

Front. Nutr. 8:739850.

doi: 10.3389/fnut.2021.739850

\section{Sex-Specific Differences in the Associations Between Omega-6 Polyunsaturated Fatty Acids and Type 2 Diabetes in Chinese People}

\author{
Yingying $\mathrm{Li}^{1}$, Hui Shen ${ }^{1}$, Yike $\mathrm{Li}^{1}$, Mei $\mathrm{Bi}^{1}$, Yanhong $\mathrm{Bi}^{2}$, Xiaoyu $\mathrm{Che}^{2}$, Simiao Tian ${ }^{2 \star}$ and \\ Yazhuo Liu ${ }^{1 *}$ \\ 1 Department of Clinical Nutrition and Metabolism, Affiliated Zhongshan Hospital of Dalian University, Dalian, China, \\ ${ }^{2}$ Department of Research, Affiliated Zhongshan Hospital of Dalian University, Dalian, China
}

Background: Some evidence indicates a potential beneficial effect of omega- 6 polyunsaturated fatty acids ( $n-6$ PUFAs) on type 2 diabetes mellitus (T2DM); however, the findings to date remains inconclusive and little is known about whether sex modifies these associations. Therefore, this study aimed to investigate potential sex-specific differences in this associations among Chinese adults.

Methods: We conducted a cross-sectional study in an area of Dalian city, China; Chinese men and women who attended the Department of Clinical Nutrition and Metabolism between January and December 2020 were invited to participate in this study. All participants were assessed for basic demographic characteristics, fasting blood glucose, HbA1c, and other serum biomarkers and serum phospholipid FAs.

Results: In total, 575 Chinese adult participants (270 men and 305 women) were included in the analysis. Hypertension and dyslipidaemia were more common among men than women, but there were no significant differences between the sexes in fatty acid composition, except for eicosadienoic acid (EA; 20:2n-6) and total monounsaturated fatty acids (MUFA). The age-adjusted OR for having T2DM in the highest quartile of arachidonic acid (20:4n-6) level was $0.47(95 \% \mathrm{Cl}, 0.22,0.98)$ in men, and this association remained consistently significant in the fully adjusted multivariate models. In contrast, no significant associations between n-6 PUFAs and T2DM risk were observed in women, regardless of model adjustment.

Conclusions: In conclusion, these results demonstrate a notable sex-specific differences in the associations between n-6 PUFAs and T2DM. Higher n-6 PUFA status may be protective against the risk of T2DM in men.

Keywords: omega- 6 fatty acids, type 2 diabetes mellitus, sex-specific, arachidonic acid, n-6 PUFAs

\section{INTRODUCTION}

Diabetes has becoming one of the most important public health issues because of its increasing prevalence worldwide and its significant clinical complications (1). In 2014, an estimated 422 million people were diagnosed with diabetes globally (2), and that number is estimated to increase to 642 million by 2040 (3). Among the general Chinese adult population, the estimated number of 
adults with diabetes in 2007-2008 was 92.4 million (4) and this increased to 164 million in 2019 (5). Diabetes is a wellrecognised risk factor for premature morbidity and mortality and affects all segments of the population (6). More importantly, it is estimated to be the seventh leading cause of death (7), and its related health complications are estimated to contribute to $\sim 0.84$ million deaths in China by 2030 (8). Therefore, appropriate treatment, lifestyle, and in particular, dietary strategies should be implemented to treat and prevent the onset of T2DM.

Among the various dietary factors, it has been suggested that n-6 PUFAs, particularly linoleic acid (18:2n-6) and arachidonic acid (20:4n-6), which can be found in plant and vegetable oils, may have potentially beneficial effect on T2DM and insulin sensitivity $(9,10)$; however, the findings related to $\mathrm{n}$ 6 biomarkers, metabolic dysfunction and health outcomes are mixed and inconclusive. Linoleic acid is the predominant $n-6$ PUFA and accounts for $80-90 \%$ of total dietary PUFAs (11), whereas arachidonic acid accounts for a minor component of total dietary PUFAs and can be obtained by means of dietary intake and the endogenous formation of linoleic acid (12). Early studies have suggested critical pro-inflammatory roles of linoleic acid and arachidonic acid (13); however, growing evidence indicates that n-6 and its main PUFA biomarkers are associated with a lower risk of T2DM, cardiometabolic diseases and their related biomarkers, including glycaemia and insulin resistance $(14,15)$. Recently, a large pooled analysis of 20 prospective cohort studies from 10 countries revealed that subjects in the highest quintile for the linoleic acid had a 35\% lower risk of T2DM when compared with subjects the lowest quintile (16), and more importantly, the protective effect of linoleic acid was generally similar in phospholipids, plasma, cholesterol esters, and adipose tissue. However, another very extensive systematic review which included only randomised controlled trials (RCT) found little to no effect of n-6 supplementation on glucose metabolism and treatment of T2DM (9). Given these inconsistent findings, the association between n-6 PUFA biomarkers and T2DM remains inconclusive.

In addition, sex may serve as an important modifying variable in the relationship between n-6 PUFAs and T2DM risk because there are clear sex-specific disparities in dietary intake habits, which lead to varying n-6 PUFA profiles (17). To date, to the best of our knowledge, there are no studies investigating the potential link between T2DM and n-6 PUFAs in serum phospholipids in the Chinese population; there are also non-studies of whether the degree and direction of the relationships between n-6 PUFAs and T2DM differ by sex. Therefore, the aim of this study was to investigate potential sex differences in the associations between n-6 PUFAs and risk of T2DM among Chinese adults.

\section{MATERIALS AND METHODS Study Population}

This was a single centre observational study performed at the Affiliated Zhongshan Hospital of Dalian University, located in Dalian, China. The Chinese patients aged $\geq 18$ years who attended the Department of Clinical Nutrition and Metabolism between January and December 2020 were invited to participate in the study. The study protocol was approved by the Institutional Ethics Committee of the Affiliated Zhongshan Hospital of Dalian University and conducted according to the Declaration of Helsinki. All methods were performed in accordance with the relevant guidelines and regulations. Written informed consent was obtained for analysis of demographics, medical histories and serum samples. Participants were invited if (1) they are Chinese people by ancestry, and lived in China for at least 5 years, (2) they were aged $\geq 18$ years and (3) willing to participate in this survey. Of 719 participants invited, we excluded participants who were diagnosed with type 1 diabetes; those who were pregnant or lactating; those who reported a history of cardiovascular disease, cancer, mentally disabled, severe liver or kidney dysfunction; those who received lipid-lowering drugs in the long-term (such as statins and fibrates); and those who received a medically supervised diet program. Of the remaining 633 participants, we further excluded 58 participants who had no available measurement of either plasma glucose or glycated heamoglobin Alc $\left(\mathrm{HbA}_{1 \mathrm{C}}\right)$, or had no complete data on serum phospholipid FA. Finally, 575 participants consented to and completed in the present study, and among them, main co-morbidities included metabolic abnormalities such as increased blood pressure (BP), impaired lipid profiles and reduced bone mineral density related to vitamin $\mathrm{D}$ deficiency.

\section{Data Collection}

Participants' demographic information was collected by a standardised questionnaire which was completed by the participants during face-to-face interviews with well-trained personnel. The standardised questionnaire included information on (1) sociodemographic characteristics such as age, gender, marital status and education level; (2) behavioural factors such as smoking status, alcohol intake and physical activity; and (3) self-reported family history. Smoking status was defined as "Yes" (current or former) if the participant smoked at least one cigarette a day for more than 6 months; alcohol intake was defined as "Yes" (current or former) if the participant had a drink at least once a week on average.

Body weight and height were measured while the participants were barefoot and dressed in light clothing: weight and height was measured by digital weighing scale (BF-220, TANITA, Tokyo, Japan) and a wall-mounted stadiometer, and taken to the nearest $0.1 \mathrm{~kg}$ and $0.1 \mathrm{~cm}$, respectively. Waist circumference was measured midway between the lowest rib and the iliac crest using a flexible anthropometric tape on the horizontal plane with the participant in the standing position; waist circumference was measured to the nearest $0.1 \mathrm{~cm}$. Body mass index (BMI) was calculated by dividing the participant's weight in kilogrammes by the square of their height in metres. Systolic and diastolic BPs (SBP and DBP) were measured on the right arm using a validated semiautomatic oscillometer (OMRON Model1 Plus; Omron Company, Kyoto, Japan). Measurements were collected in triplicate after seated rest for $10 \mathrm{~min}$; the mean of the three measurements was used in the analyses.

Fasting blood samples were collected in the morning following an overnight fast. Plasma and serum samples were then frozen and stored at $-80^{\circ} \mathrm{C}$ for later laboratory analysis. Serum 
level of fasting plasma glucose (FPG) was measured using the hexokinase glucose-6-phosphate dehydrogenase method (ADVIA1650 Chemistry System, Bayer, Leverkusen, Germany), and the fasting serum lipid profiles such as total cholesterol (TC), high-density lipoprotein cholesterol (HDL-C) concentration, triglycerides (TG) were measured by enzymatic colorimetric method (HITACHI-7170, Hitachi, Tokyo, Japan). In addition, $\mathrm{HbA}_{1 \mathrm{c}}$ was determined by the method established by the highperformance liquid chromatography (HPLC) method (BIORAD, Hercules, CA, USA). All blood samples were measured at a central, certified laboratory in Dalian with strict quality control.

\section{Measurement of FA Composition}

Serum FAs were measured from samples stored at $-80^{\circ} \mathrm{C}$ using gas chromatography by means of an AB SCIEX Triple Quad 4500MD liquid chromatography-mass spectrometery (LCMS4500MD) held at the Institute of Metal Research Chinese Academy of Sciences (located at Dalian city). A detailed description of the laboratory analyses has been provided previously (18). In brief, serum FAs were extracted from whole blood using the Folch et al. method (19) with chloroformmethanol (2:1). The chloroform phase was evaporated and treated with sodium methoxide, which methylated the esterified fatty acids. The oven temperature increased by $5^{\circ} \mathrm{C} / \mathrm{min}$ from $140^{\circ} \mathrm{C}$ to $220^{\circ} \mathrm{C}$ during the analysis run. The peaks were identified on the basis of retention times recorded for different standards. FA levels were quantified by gas-liquid chromatography with reference standards purchased from NUCheque Prep, Inc, and each analyte had an individual reference standard. The FAs were expressed as as absolute concentrations in serum $(\mathrm{nmol} / \mathrm{ml})$ based on the quantity of eicosane used as an internal standard. The intra- and inter-assay coefficients of variation $(\mathrm{CV}) \%$ for individual FAs, which were calculated using two serum samples as quality controls added to each batch, were $\leq 10$ and $\leq 12 \%$, respectively.

\section{Ascertainment of Outcome Variable T2DM}

Participants were defined as having T2DM if their $\mathrm{HbA}_{1 \mathrm{C}}$ levels exceeded $6.5 \%$ or if they were currently under medical treatment for T2DM according to the American Diabetes Association criteria for the diagnosis of diabetes, regardless of fasting glucose levels (20).

\section{Definition of Exposure Variables}

In this study, patients were diagnosed with hypertension if they had a systolic blood pressure of $\geq 140 \mathrm{mmHg}$ and/or a diastolic blood pressure of $\geq 90 \mathrm{mmHg}$ or if they selfreported use of antihypertensive medication (21). Patients were diagnosed with dyslipidemia were diagnosed if they fulfilled the following criteria: LDL-cholesterol $\geq 4.14 \mathrm{mmol} / \mathrm{L}$, HDLcholesterol $<1.036 \mathrm{mmol} / \mathrm{L}$ and triglycerides $\geq 2.26 \mathrm{mmol} / \mathrm{L}$ (22).

\section{Statistical Analyses}

The characteristics of the study population were presented as the mean and SD or median (interquartile range; IQR) for continuous variables, where appropriate, and as numbers and percentages for categorical variables. Comparisons between two sex groups were assessed by using Student's $t$-test or the Mann Whitney- $U$-test for continuous variables, and chisquare tests for categorical variables. In the examination of bivariate relationships between serum phospholipid n-6 PUFAs and T2DM biomarkers (e.g., FPG and HbA1C), the analysis was performed separately by sex and Spearman partial correlations were used, adjusting for age and BMI. Multivariate logistic regression analysis was conducted to estimate the associations between T2DM and both total and individual n-6 PUFAs, as well as other FAs, with odds ratios (ORs) and $95 \%$ confidence intervals (CIs) calculated. This analysis was also performed separately by sex and several different models with sequential adjustments for key potential confounders were fitted. Model 1 was adjusted for age; Model 2 was additionally adjusted for smoking status, alcohol status and BMI (model 2); and Model 3 was further adjusted for hypertension and dyslipidaemia. All statistical analyses were performed using $\mathrm{R}$ version 3.6 .3 software (23). A $P$-value $<0.05$ was considered statistically significant.

\section{RESULTS}

In this study, a final sample included 575 adult participants (270 men and 305 women) with a mean age of $58.23 \pm$ 11.94 years. There were significant differences between men and women in several anthropometric and biochemical variables (Table 1). Men had significantly higher BP, FPG, TG and UA levels compared to women, whereas they had lower TC, HDL$\mathrm{C}$ and LDL-C levels than women. With regard to circulating serum fatty acid composition, no significant differences were observed between men and women among all except EA and total MUFA. Moreover, smoking and alcohol intake were more prevalent among men than women (smoking: 44.4 vs. $1.31 \%$; alcohol intake: 40 vs. $4.92 \%$, respectively). Hypertension and dyslipidaemia were more common among men than women, with both prevalent among more $40 \%$ of men and nearly $30 \%$ of women, respectively.

The overall and sex-specific distributions of serum FA compositions between participants with and without diabetes are shown in Table 2. Among overall population, none of these FA compositions showed any significant differences between two diabetic status; however, participants with T2DM had lower levels of total n-3 PUFAs, n-6 PUFAs, and PUFAs, and arachidonic acid compared with those without T2DM, in contrast a slight higher levels of linoleic acid, EA, total saturated fatty acids (SFAs) and MUFAs was found in participants with diabetes. With regard to men, a consistent lower levels of serum FA compositions was noted in men with diabetes when compared to their peers without, whereas for women, a contrast pattern was found such that women with diabetes had a slight higher levels of each FA composition than women without diabetes.

Spearman partial correlations between each serum FA biomarker and $\mathrm{FPG}$ and $\mathrm{HbA}_{1 \mathrm{C}}$ were calculated after adjusting for age and BMI, in both the overall sample and sex-specific samples (Table 3). Among the total sample, there were weak but significant positive correlations between $\mathrm{HbA}_{1 \mathrm{C}}$ and all except linoleic acid and EA; however, these correlations became 
TABLE 1 | Basic characteristics of studied sample.

\begin{tabular}{|c|c|c|c|c|}
\hline & Total sample $(n=575)$ & Men $(n=270)$ & Women $(n=305)$ & $P$-value \\
\hline Age, years & $58.23 \pm 11.94$ & $58.06 \pm 12.31$ & $58.38 \pm 11.62$ & 0.748 \\
\hline Smoker, $n(\%)$ & 124 (21.57\%) & $120(44.44 \%)$ & $4(1.31 \%)$ & $<0.001$ \\
\hline Alcohol drinker, $n$ (\%) & $123(21.39 \%)$ & 108 (40.00\%) & $15(4.92 \%)$ & $<0.001$ \\
\hline Weight, kg & $70.47 \pm 12.85$ & $77.88 \pm 11.78$ & $63.90 \pm 9.84$ & $<0.001$ \\
\hline Height, cm & $168.32 \pm 7.68$ & $174.19 \pm 5.49$ & $163.12 \pm 5.18$ & $<0.001$ \\
\hline BMl, kg/m² & $24.76 \pm 3.43$ & $25.62 \pm 3.35$ & $23.99 \pm 3.33$ & $<0.001$ \\
\hline SBP, mm Hg & $130.91 \pm 19.18$ & $133.93 \pm 19.61$ & $128.24 \pm 18.41$ & $<0.001$ \\
\hline DBP, mm Hg & $81.78 \pm 10.61$ & $84.07 \pm 10.61$ & $79.76 \pm 10.22$ & $<0.001$ \\
\hline FPG, mmol/L & $6.01 \pm 2.57$ & $6.15 \pm 2.30$ & $5.89 \pm 2.79$ & 0.219 \\
\hline $\mathrm{HbA}_{1 \mathrm{C}}, \%$ & $6.27 \pm 1.48$ & $6.38 \pm 1.54$ & $6.16 \pm 1.43$ & 0.081 \\
\hline $\mathrm{TC}, \mathrm{mmol} / \mathrm{L}$ & $5.18 \pm 1.09$ & $4.97 \pm 1.04$ & $5.37 \pm 1.11$ & $<0.001$ \\
\hline HDL-C, mmol/L & $1.44 \pm 0.45$ & $1.33 \pm 0.49$ & $1.55 \pm 0.38$ & $<0.001$ \\
\hline LDL-C, mmol/L & $3.52 \pm 13.75$ & $2.82 \pm 0.78$ & $4.14 \pm 18.85$ & 0.223 \\
\hline $\mathrm{TG}, \mathrm{mmol} / \mathrm{L}$ & $1.82 \pm 1.27$ & $2.06 \pm 1.56$ & $1.61 \pm 0.89$ & $<0.001$ \\
\hline $\mathrm{UA}, \mu \mathrm{mol} / \mathrm{L}$ & $337.06 \pm 99.75$ & $379.79 \pm 98.44$ & $299.38 \pm 84.69$ & $<0.001$ \\
\hline Hypertension, $n$ (\%) & 224 (38.96\%) & 124 (45.93\%) & 100 (32.79\%) & 0.002 \\
\hline Dyslipidaemia, $n$ (\%) & 217 (37.74\%) & $115(42.59 \%)$ & $102(33.44 \%)$ & 0.03 \\
\hline Total SFAs & $5,961.08 \pm 2,567.60$ & $6,125.20 \pm 2789.68$ & $5,815.79 \pm 2,348.70$ & 0.154 \\
\hline Total MUFAs & $2,767.82 \pm 1,622.26$ & $2,991.01 \pm 1917.91$ & $2,570.24 \pm 1,277.03$ & 0.002 \\
\hline Total PUFAs & $7,466.41 \pm 2,420.70$ & $7,391.04 \pm 2584.75$ & $7,533.12 \pm 2,267.82$ & 0.486 \\
\hline Total n-3 PUFAs & $711.61 \pm 369.14$ & $705.54 \pm 390.65$ & $716.98 \pm 349.56$ & 0.713 \\
\hline Total n-6 PUFAs & $6,431.79 \pm 2,054.92$ & $6,358.43 \pm 2177.27$ & $6,496.72 \pm 1,941.49$ & 0.424 \\
\hline Linoleic acid (18:2n-6) & $5,227.26 \pm 1,654.26$ & $5,182.05 \pm 1784.64$ & $5,267.29 \pm 1,531.48$ & 0.542 \\
\hline Eicosadienoic acid (20:2n-6) & $31.99 \pm 15.57$ & $33.66 \pm 17.49$ & $30.51 \pm 13.49$ & 0.017 \\
\hline Arachidonic acid (20:4n-6) & $1,204.52 \pm 552.03$ & $1176.38 \pm 533.40$ & $1229.44 \pm 567.71$ & 0.249 \\
\hline
\end{tabular}

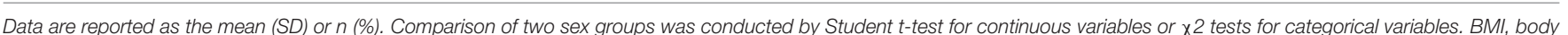

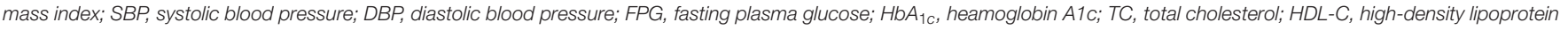

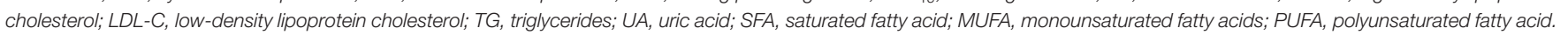

non-significant inverse for total n-6 PUFAs, linoleic acid, arachidonic acid and EA with FPG. In men, there were inverse and non-significant correlations between these FAs and FPG, and these correlations became positive for $\mathrm{HbA}_{1 \mathrm{C}}$. In women, all FAs except EA were positively correlated with both FPG and $\mathrm{HbA}_{1 \mathrm{C}}$, but the significant positive correlations were only observed between $\mathrm{HbA}_{1 \mathrm{C}}$ biomarker and FAs except total n-6 PUFAs, linoleic acid and EA in women.

The sex-specific associations between FA biomarkers and T2DM are presented in Table 4. In men, among the n-6 PUFAs, arachidonic acid was significantly and inversely associated with T2DM, with age-adjusted OR value of 0.47 (95\% CI, 0.22, 0.98) when comparing the highest quartile of arachidonic acid to the lowest (Model 1). This association was consistently significant in two fully adjusted multivariate models (Model 2 and Model 3 ). The ORs (95\% CIs) of T2DM in the model 3 for the lowest through the highest quartiles of arachidonic acid were $1.00,0.62$ $(0.3,1.28), 0.59(0.28,1.24)$, and $0.42(0.19,0.92)$, respectively. With regard to other n-6 PUFAs, higher level of linoleic acid and eicosadienoic acid were likely to reduce the chance of having T2DM compared to lower levels (i.e., lowest quartile), but none of these associations were statistically significant either in the basic age-adjusted model or the fully multivariable-adjusted model.
Furthermore, none of total n-3 PUFAs, SFAs nor MUFAs were significantly associated with T2DM, regardless of whether the model was adjusted.

In women, neither of total n-6 PUFAs nor individual n6 PUFAs were found significantly associated with T2DM, although there were inverse association patterns for linoleic acid, arachidonic acid and EA. However, compared to the lowest quartile, having T2DM was higher in the highest quartile of both SFAs and MUFAs, but these positive associations were not statistically significant; moreover, a flat J-shaped relationships for both SFAs and MUFAs seemed to be found, with a decrease in OR of T2DM in the second quartile, after which ORs moderately rose to 1.49 and 1.54 , respectively, but remaining no significant association.

\section{DISCUSSION}

In the present study, the serum concentration of arachidonic acid was inversely associated with T2DM in Chinese men but not in Chinese women, indicating a sex-specific effect in this association. There were inverse association patterns between total n-6 PUFAs, linoleic acid and T2DM; however, these associations were non-significant after adjustment for multiple confounders, 


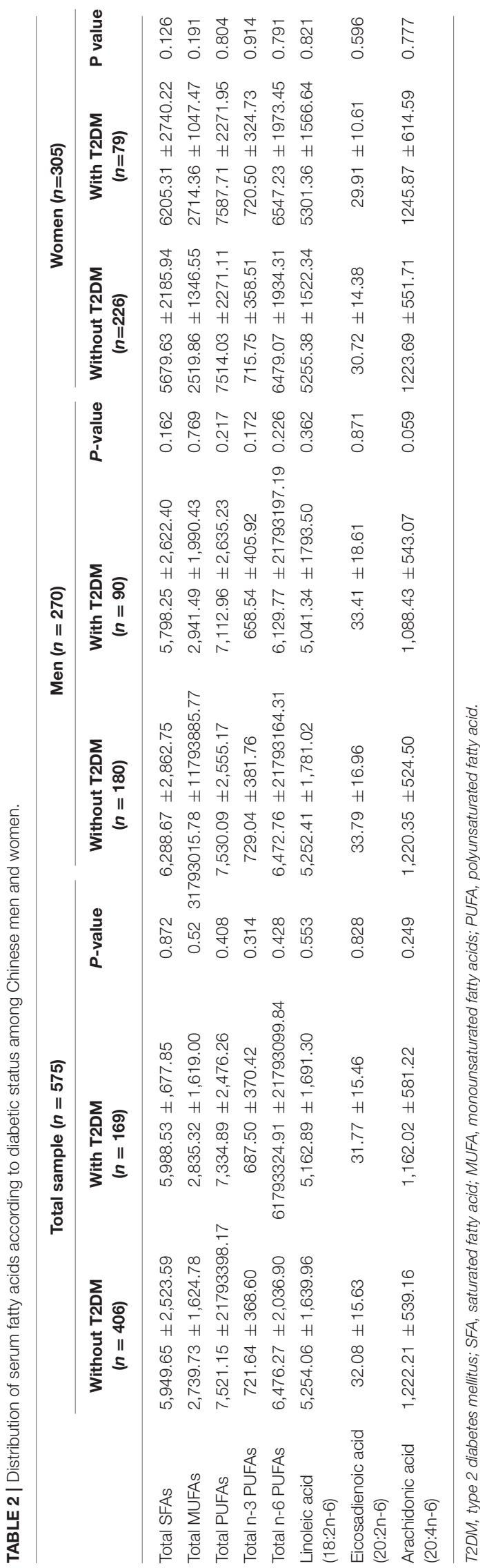

TABLE 3 | Spearman partial correlations between serum fatty acid biomarkers and $F P G$ and $\mathrm{HbA}_{1 \mathrm{C}}$ stratified by sex.

\begin{tabular}{|c|c|c|c|}
\hline & & $\begin{array}{c}\text { FPG } \\
\text { Partial-r }\end{array}$ & $\begin{array}{c}\mathrm{HbA}_{1 \mathrm{c}} \\
\text { Partial-r }\end{array}$ \\
\hline \multirow[t]{8}{*}{ Total sample } & Total SFAs & 0.016 & $0.156^{\star}$ \\
\hline & Total MUFAs & 0.052 & $0.117^{\star}$ \\
\hline & Total PUFAs & -0.024 & $0.091^{*}$ \\
\hline & Total n-3 PUFAs & 0.024 & $0.131^{\star}$ \\
\hline & Total n-6 PUFAs & -0.030 & $0.082^{*}$ \\
\hline & Linoleic acid (18:2n-6) & -0.027 & 0.070 \\
\hline & Eicosadienoic acid (20:2n-6) & -0.028 & 0.038 \\
\hline & Arachidonic acid (20:4n-6) & -0.038 & $0.104^{*}$ \\
\hline \multirow[t]{8}{*}{ Men } & Total SFAs & -0.072 & 0.107 \\
\hline & Total MUFAs & 0.008 & 0.069 \\
\hline & Total PUFAs & -0.046 & 0.098 \\
\hline & Total n-3 PUFAs & -0.001 & 0.106 \\
\hline & Total n-6 PUFAs & -0.052 & 0.097 \\
\hline & Linoleic acid (18:2n-6) & -0.047 & 0.092 \\
\hline & Eicosadienoic acid (20:2n-6) & -0.008 & 0.034 \\
\hline & Arachidonic acid (20:4n-6) & -0.081 & 0.067 \\
\hline \multirow[t]{8}{*}{ Women } & Total SFAs & $0.121^{\star}$ & $0.214^{\star}$ \\
\hline & Total MUFAs & 0.094 & $0.1771^{*}$ \\
\hline & Total PUFAs & 0.018 & 0.098 \\
\hline & Total n-3 PUFAs & 0.070 & $0.174^{*}$ \\
\hline & Total n-6 PUFAs & 0.013 & 0.083 \\
\hline & Linoleic acid (18:2n-6) & 0.011 & 0.057 \\
\hline & Eicosadienoic acid (20:2n-6) & -0.057 & 0.045 \\
\hline & Arachidonic acid (20:4n-6) & 0.018 & $0.158^{\star}$ \\
\hline
\end{tabular}

Correlations adjusted for age and BMI.

${ }^{*} P<0.05$. FPG, fasting plasma glucose; $H b A_{1 c}$, heamoglobin A1c; SFA, saturated fatty acid; MUFA, monounsaturated fatty acids; PUFA, polyunsaturated fatty acid.

regardless of sex. To the best of our knowledge, this is the first study to investigate sex-dependent associations between $\mathrm{n}-6$ PUFAs and T2DM in the Chinese population.

The current finding of a reduced change of having T2DM with a higher concentration of serum arachidonic acid is consistent with several studies in Western countries. In the KIHD (Kuopio Ischaemic Heart Disease) case-cohort study, Yary et al. reported significant inverse associations between the risk of incident T2DM and both serum arachidonic acid and the D5D, a measure of arachidonic acid-based desaturase activity, in a 19-y followup study (15). In another prospective cohort study comprising 407 overweight, middle-aged men and women, Takkunen et al. found a very similar result that both serum arachidonic acid and D5D were significantly and inversely associated with the risk of $\mathrm{T} 2 \mathrm{DM}$, and for every one-unit increase in serum arachidonic acid, there was a $20 \%$ lower incidence of T2DM during the median follow-up of 11 years (18). The present findings are also consistent with several Asian studies and meta-analyses, although most did not find significant associations. For example, in a prospective study of Japanese employees, the incidence of T2DM exhibited a non-significant decreasing trend with elevated circulating serum arachidonic acid (24). Consistently, 
TABLE 4 | Association between serum FAs and risk of type 2 diabetes mellitus stratified by Chinese men and women.

\begin{tabular}{|c|c|c|c|c|c|c|c|c|c|}
\hline & \multirow[t]{2}{*}{ Model } & \multicolumn{4}{|c|}{ Men } & \multicolumn{4}{|c|}{ Women } \\
\hline & & 1st Quartile & 2nd Quartile & 3rd Quartile & 4th Quartile & 1st Quartile & 2nd Quartile & 3rd Quartile & 4th Quartile \\
\hline \multirow[t]{3}{*}{ Total SFAs } & Model 1 & 1 (reference) & $0.71(0.35,1.46)$ & $1.09(0.51,2.33)$ & $0.58(0.28,1.22)$ & 1 (reference) & $0.77(0.33,1.77)$ & $1.35(0.66,2.76)$ & $1.62(0.76,3.44)$ \\
\hline & Model 2 & 1 (reference) & $0.70(0.34,1.45)$ & $1.00(0.46,2.17)$ & $0.53(0.25,1.12)$ & 1 (reference) & $0.71(0.30,1.68)$ & $1.28(0.61,2.69)$ & $1.42(0.65,3.10)$ \\
\hline & Model 3 & 1 (reference) & $0.70(0.33,1.46)$ & $0.93(0.42,2.05)$ & $0.49(0.22,1.10)$ & 1 (reference) & $0.74(0.31,1.78)$ & $1.26(0.59,2.71)$ & $1.49(0.63,3.52)$ \\
\hline \multirow[t]{3}{*}{ Total MUFAs } & Model 1 & 1 (reference) & $0.67(0.32,1.43)$ & $0.86(0.41,1.80)$ & $0.67(0.32,1.42)$ & 1 (reference) & $0.61(0.28,1.34)$ & $0.79(0.37,1.67)$ & $1.57(0.76,3.25)$ \\
\hline & Model 2 & 1 (reference) & $0.64(0.30,1.37)$ & $0.78(0.37,1.65)$ & $0.58(0.27,1.26)$ & 1 (reference) & $0.58(0.25,1.31)$ & $0.68(0.31,1.47)$ & $1.39(0.65,2.95)$ \\
\hline & Model 3 & 1 (reference) & $0.64(0.30,1.39)$ & $0.73(0.33,1.58)$ & $0.48(0.20,1.19)$ & 1 (reference) & $0.56(0.24,1.28)$ & $0.68(0.30,1.52)$ & $1.54(0.64,3.72)$ \\
\hline \multirow[t]{3}{*}{ Total PUFAs } & Model 1 & 1 (reference) & $0.87(0.43,1.75)$ & $0.67(0.32,1.42)$ & $0.76(0.36,1.59)$ & 1 (reference) & $1.04(0.47,2.30)$ & $1.08(0.50,2.33)$ & $1.22(0.57,2.61)$ \\
\hline & Model 2 & 1 (reference) & $0.86(0.42,1.74)$ & $0.60(0.28,1.30)$ & $0.73(0.35,1.56)$ & 1 (reference) & $1.14(0.50,2.61)$ & $1.05(0.47,2.32)$ & $1.26(0.57,2.77)$ \\
\hline & Model 3 & 1 (reference) & $0.85(0.41,1.73)$ & $0.57(0.26,1.26)$ & $0.66(0.30,1.49)$ & 1 (reference) & $1.08(0.47,2.50)$ & $0.97(0.42,2.24)$ & $1.19(0.51,2.76)$ \\
\hline \multirow[t]{3}{*}{ Total n-3 PUFAs } & Model 1 & 1 (reference) & $1.04(0.51,2.13)$ & $0.42(0.19,0.92)$ & $0.85(0.41,1.75)$ & 1 (reference) & $0.83(0.38,1.83)$ & $1.07(0.50,2.29)$ & $1.11(0.52,2.37)$ \\
\hline & Model 2 & 1 (reference) & $0.93(0.45,1.93)$ & $0.39(0.17,0.87)$ & $0.79(0.38,1.64)$ & 1 (reference) & $0.85(0.37,1.92)$ & $0.95(0.43,2.10)$ & $1.08(0.49,2.38)$ \\
\hline & Model 3 & 1 (reference) & $0.92(0.44,1.91)$ & $0.40(0.18,0.89)$ & $0.75(0.35,1.61)$ & 1 (reference) & $0.84(0.37,1.91)$ & $0.94(0.42,2.13)$ & $1.05(0.45,2.45)$ \\
\hline \multirow[t]{3}{*}{ Total n-6 PUFAs } & Model 1 & 1 (reference) & $0.85(0.42,1.73)$ & $0.99(0.49,2.00)$ & $0.69(0.32,1.49)$ & 1 (reference) & $1.16(0.52,2.57)$ & $1.22(0.56,2.68)$ & $1.31(0.61,2.82)$ \\
\hline & Model 2 & 1 (reference) & $0.81(0.39,1.67)$ & $0.90(0.44,1.85)$ & $0.69(0.31,1.50)$ & 1 (reference) & $1.12(0.49,2.55)$ & $1.13(0.50,2.53)$ & $1.30(0.59,2.85)$ \\
\hline & Model 3 & 1 (reference) & $0.82(0.40,1.71)$ & $0.85(0.41,1.79)$ & $0.64(0.28,1.46)$ & 1 (reference) & $1.07(0.47,2.45)$ & $1.06(0.45,2.49)$ & $1.23(0.54,2.83)$ \\
\hline \multirow[t]{3}{*}{ Linoleic acid (18:2n-6) } & Model 1 & 1 (reference) & $0.98(0.48,2.00)$ & $1.10(0.54,2.22)$ & $0.83(0.39,1.78)$ & 1 (reference) & $0.79(0.36,1.77)$ & $1.26(0.59,2.69)$ & $0.99(0.46,2.10)$ \\
\hline & Model 2 & 1 (reference) & $0.94(0.46,1.95)$ & $1.00(0.48,2.05)$ & $0.82(0.38,1.78)$ & 1 (reference) & $0.77(0.34,1.75)$ & $1.24(0.57,2.69)$ & $0.95(0.43,2.06)$ \\
\hline & Model 3 & 1 (reference) & $0.92(0.44,1.91)$ & $0.93(0.44,1.96)$ & $0.76(0.34,1.74)$ & 1 (reference) & $0.72(0.31,1.66)$ & $1.20(0.54,2.68)$ & $0.92(0.40,2.09)$ \\
\hline \multirow[t]{3}{*}{ Eicosadienoic acid (20:2n-6) } & Model 1 & 1 (reference) & $1.35(0.62,2.92)$ & $1.60(0.75,3.38)$ & $1.12(0.52,2.40)$ & 1 (reference) & $0.84(0.40,1.75)$ & $0.83(0.40,1.71)$ & $0.75(0.35,1.60)$ \\
\hline & Model 2 & 1 (reference) & $1.24(0.56,2.74)$ & $1.57(0.73,3.35)$ & $1.02(0.46,2.24)$ & 1 (reference) & $0.84(0.39,1.79)$ & $0.86(0.41,1.82)$ & $0.68(0.31,1.52)$ \\
\hline & Model 3 & 1 (reference) & $1.20(0.54,2.67)$ & $1.57(0.71,3.43)$ & $0.96(0.41,2.25)$ & 1 (reference) & $0.78(0.36,1.68)$ & $0.77(0.35,1.68)$ & $0.64(0.27,1.50)$ \\
\hline \multirow[t]{3}{*}{ Arachidonic acid (20:4n-6) } & Model 1 & 1 (reference) & $0.63(0.31,1.28)$ & $0.59(0.29,1.23)$ & $0.47(0.22,0.98)$ & 1 (reference) & $1.10(0.50,2.43)$ & $1.37(0.64,2.92)$ & $0.93(0.42,2.08)$ \\
\hline & Model 2 & 1 (reference) & $0.62(0.30,1.26)$ & $0.59(0.28,1.24)$ & $0.44(0.21,0.94)$ & 1 (reference) & $1.15(0.51,2.61)$ & $1.35(0.62,2.96)$ & $0.97(0.42,2.20)$ \\
\hline & Model 3 & 1 (reference) & $0.62(0.30,1.28)$ & $0.59(0.28,1.24)$ & $0.42(0.19,0.92)$ & 1 (reference) & $1.07(0.47,2.46)$ & $1.27(0.58,2.82)$ & $0.92(0.40,2.14)$ \\
\hline
\end{tabular}

Values are ORs (95\% Cls) by quartile unless otherwise indicated. SFA, saturated fatty acid; MUFA, monounsaturated fatty acids; PUFA, polyunsaturated fatty acid. Model 1: adjusted for age; Model 2: Adjusted for Model $1+$ smoking status, alcohol status and BMI; Model 3: Adjusted for Model 2 + hypertension and dyslipidaemia. The bold values mean the results are significant. 
in a recent meta-analysis which pooled 39,740 adults from 20 prospective cohort studies (16), higher arachidonic acid was inversely associated with T2DM risk, but this was only statistically significant in plasma; the authors concluded that independent of lipid compartments, higher arachidonic acid does not contribute to T2DM development.

Arachidonic acid is a proinflammatory marker of glucose metabolism and weight regulation (25); thus, higher arachidonic acid may lead to an increased risk of T2DM from a theorectical perspective. However, the current study and several abovementioned studies suggested an inverse association with T2DM, and several mechanisms behind these associations have been suggested. For instance, arachidonic acid is a precursor to several anti-inflammatory metabolites (26) and a systematic review provided solid evidence indicating that higher concentrations of arachidonic acid are associated with a lower risk of coronary heart disease incidence (27). Therefore, the association between arachidonic acid and disease risk is difficult to predict solely on the basis of its effects on proinflammatory factors. Moreover, previous experimental studies have reported a protective effect of arachidonic acid on pancreatic $b$ cells in a chemical-induced cytotoxicity model; this effect was related to increased antioxidant status and suppression of cytokine production $(28,29)$. This above mechanism emphasises that arachidonic acid could preserve insulin production capacity, meaning that the inverse association observed in this study is biologically plausible.

Several prospective and observational studies have reported relationships between PUFAs and risk of T2DM, but few studies have focused on sex-specific differences in these associations. Abbott et al. were among the first to address the sex differences in the associations between PUFA biomarkers and T2DM among the Western population (30). Further, in the RHLS (Retirement Health and Lifestyle Study) (31) and a meta-analysis of RCTs (32), the authors reported significant inverse relationships between n3 PUFAs and both insulin resistance and T2DM in women but not men, indicating a clear sex-specific difference and suggesting a protective effect of n-3 PUFAs on insulin resistance and $\mathrm{T} 2 \mathrm{DM}$ in the female population. However, in a prospective cohort comprising only 2189 middle-aged and older Finnish men, Yary et al. found that serum n-6 PUFAs, including linoleic acid and arachidonic acid, were inversely associated with the risk of incident T2DM (15); compared to the lowest quartile, men in the highest serum linoleic acid and arachidonic acid quartiles had a 48 and 38\% lower hazard ratio of incident T2DM, respectively (15). These findings are consistent with the current study where that arachidonic acid was inversely associated with T2DM in men but not in women. The mechanisms by which n-6 PUFAs reduce the chance of having T2DM in men but not in women are not fully understood, but several have been suggested, including sex differences in n-6 PUFA distribution (33), exogenous dietary intake (34), and sex hormone-mediated adipose tissue distribution $(35,36)$. Further research is required to investigate the mechanisms underlying the sex-dependent association observed in this study.

Several prospective, observational and meta-analysis studies among Western and Asian populations have investigated the relationships between concentrations of total n-6 PUFAs, linoleic acid and T2DM risk, but the findings are conflicting; some have reported significant inverse associations $(14,16,18,37)$, whereas others have not $(24,38)$. In the EPIC (European Prospective Investigation into Cancer and Nutrition)-InterAct study, Forouhi et al. provided robust evidence of strong and significant inverse associations between T2DM and both total circulating plasma n-6 PUFAs and linoleic acid; the risk decreased by 13 and $20 \%$ for every 1-SD increase, respectively (14). Consistently, in the above-mentioned recent meta-analysis (16) and another prospective study comprising three large American cohorts (39), higher concentrations of total blood n-6 PUFAs and linoleic acid were associated with a lower risk of T2DM, independent of lipid compartment. However, the current study observed non-significant inverse relationships between T2DM and both total n-6 PUFAs and linoleic acid. In line with the current findings, several other studies have found similar but nonsignificant association patterns between T2DM and total n6 PUFAs (24) and linoleic acid $(24,40)$ after adjustment for confounders, especially BMI. For instance, in a prospective study in the Japanese population, higher concentrations of total n-6 PUFAs and linoleic acid were found to be significantly associated a lower risk of T2DM before BMI adjustment, but these associations were moderately attenuated and became non-significant when adjusting for BMI. Similarly, in another multi-ethnic atherosclerosis study, although a significant inverse association was observed between linoleic acid and T2DM risk among the total sample, the association was non-significant among the subgroup of Chinese people (37). It can be speculated that the reasons for these differences may be due to differences in major dietary sources of n-6 PUFAs and linoleic acid. Considering the potential weight-reducing effect of LA (41), it may have long-term benefits in preventing T2DM risk, at least in part, by means of its effect on body fat.

In addition to studying the risks of endogenous PUFAs on T2DM, exogenous supplementary PUFAs are often used clinically. Many studies have investigated the role of intake of various FAs in T2DM prevention; for instance, higher intake of PUFA (42), n-6 FAs $(9,39)$ and n-3 FAs (42), as well as lower intake of saturated FAs (43) and trans-fatty acids (42), is recommended. However, the role of endogenous intake of PUFAs remains controversial. Of note, few prospective and RCT studies have focused on n-6 intake for T2DM prevention $(39,44-$ 48 ), and the majority observed a null or very week associations between T2DM and n-6 FA intake, with relative risk values ranging from 0.97 to 1.01 . In a very recent dose-response metaanalysis, Neuenschwander et al. reported null findings with regard to the associations between T2DM and intake of total n-6 and linoleic acid (49). This finding is inconsistent with another recent meta-analysis of RCTs (9), in which the authors suggested a favourable effect of higher n-6 FA intake in T2DM prevention. These discrepancies, as Neuenschwander et al. argue, may be due to the very limited number of included trials (only 2 trials) and the variation in the control groups from Brown et al. meta-analysis study, which could attenuate the strength of the evidence. Together with the findings from previous prospective and interventional studies of T2DM, the current findings do not 
suggest that high levels of n-6 PUFAs are harmful; however, given the inconsistent data, more research is warranted to clarify the associations between n-6 PUFAs, linoleic acid and T2DM.

The notable strengths of this study should be mentioned. First, assessment for diabetes was based on objective measurements including $\mathrm{HbA}_{1 \mathrm{c}}$ and blood glucose as well as self-report data. Second, FA metabolism profile was determined using liquidphase tandem mass spectrometry, which guarantees the rigorous quality of the data. Nonetheless, this study also has several limitations that should be acknowledged. First, due to the nature of the cross-sectional design, this study could only examine the associations and related magnitude between n- 6 PUFAs and T2DM and the causality could not be determined. Cohort studies are warranted to clarify the current findings. Second, despite study sample of inpatients, the participants with unstable clinical situations or severe diseases were excluded to minimise the sample bias. Meanwhile, the analyses were adjusted for multiple potential confounders and co-morbidities to minimise another possibility of bias. Third, there was a low sample size of female smoker and drinker in the studied sample, and larger sample size for these subsample will be considered for the further study. In addition, the sample included only Chinese subjects; thus, generalisability of the results to a wider population should be undertaken with caution.

\section{CONCLUSION}

In conclusion, a higher serum concentration of arachidonic acid was associated with a lower change of having T2DM among Chinese men. In addition, the current results suggest sex-specific differences in the association between n-6 PUFAs and T2DM in Chinese people, and a favourable impact of arachidonic acid on T2DM, especially in Chinese men. Further research is needed to elucidate the sex effects on PUFA metabolism and the mechanisms involved.

\section{REFERENCES}

1. Lam DW, LeRoith D. The worldwide diabetes epidemic. Curr Opin Endocrinol Diabetes Obes. (2012) 19:93-6. doi: 10.1097/MED.0b013e3283 $50583 \mathrm{a}$

2. Lovic D, Piperidou A, Zografou I, Grassos H, Pittaras A, Manolis A. The growing epidemic of diabetes mellitus. Curr Vasc Pharmacol. (2020) 18:104-9. doi: 10.2174/157016111766619040 5165911

3. Ogurtsova K, da Rocha Fernandes JD, Huang Y, Linnenkamp U, Guariguata L, Cho $\mathrm{NH}$, et al. IDF diabetes atlas: global estimates for the prevalence of diabetes for 2015 and 2040. Diabetes Res Clin Pract. (2017) 128:40-50. doi: 10.1016/j.diabres.2017. 03.024

4. Yang W, Lu J, Weng J, Jia W, Ji L, Xiao J, et al. Prevalence of diabetes among men and women in China. N Engl J Med. (2010) 362:1090101. doi: 10.1056/NEJMoa0908292

5. Luo Z, Fabre G, Rodwin VG. Meeting the challenge of diabetes in China. Int J Health Policy Manag. (2020) 9:47-52. doi: 10.15171/ijhpm. 2019.80

6. Magliano DJ, Sacre JW, Harding JL, Gregg EW, Zimmet PZ, Shaw JE. Young-onset type 2 diabetes mellitus - implications for morbidity and

\section{DATA AVAILABILITY STATEMENT}

The datasets used and analyzed during the current study available from the corresponding authors on reasonable request.

\section{ETHICS STATEMENT}

The studies involving human participants were reviewed and approved by Ethics Committee of the Affiliated Zhongshan Hospital of Dalian University. The patients/participants provided their written informed consent to participate in this study.

\section{AUTHOR CONTRIBUTIONS}

YaL and ST: conceptualization and supervision. MB: data acquisition. YB and XC: statistical analysis. ST and YaL: investigation and writing-review and editing. YinL, HS, and YikL: writing-original draught. All authors have read and agreed to the published version of the manuscript.

\section{FUNDING}

This study was supported by National Natural Science Foundation of China (81803329). The funders of the study had no role in the data collection and analysis, interpretation of this work, or the decision to submit this work for publication.

\section{ACKNOWLEDGMENTS}

The authors would like to acknowledge all the staff of the Department of Clinical Nutrition and Metabolism at affiliated Zhongshan hospital of Dalian University for their contributions, and all the subjects participating in this study.

mortality. Nat Rev Endocrinol. (2020) 16:321-31. doi: 10.1038/s41574-0200334-z

7. Carley AN, Kleinfeld AM. Fatty acid (FFA) transport in cardiomyocytes revealed by imaging unbound FFA is mediated by an FFA pump modulated by the CD36 protein. J Biol Chem. (2011) 286:4589-97. doi: 10.1074/jbc.M110.182162

8. Wang L, Gao P, Zhang M, Huang Z, Zhang D, Deng Q, et al. Prevalence and ethnic pattern of diabetes and prediabetes in China in 2013. JAMA. (2017) 317:2515-23. doi: 10.1001/jama.2017.7596

9. Brown TJ, Brainard J, Song F, Wang X, Abdelhamid A, Hooper L, et al. Omega-3, omega-6, and total dietary polyunsaturated fat for prevention and treatment of type 2 diabetes mellitus: systematic review and metaanalysis of randomised controlled trials. BMJ. (2019) 366:14697. doi: 10.1136/ bmj.14697

10. Schwab U, Lauritzen L, Tholstrup T, Haldorssoni T, Riserus U, Uusitupa M, et al. Effect of the amount and type of dietary fat on cardiometabolic risk factors and risk of developing type 2 diabetes, cardiovascular diseases, and cancer: a systematic review. Food Nutr Res. (2014) 58:25145. doi: 10.3402/fnr.v58.25145

11. Micha R, Khatibzadeh S, Shi P, Fahimi S, Lim S, Andrews KG, et al. Global, regional, and national consumption levels of dietary fats and oils in 1990 and 2010: a systematic analysis including 266 country-specific nutrition surveys. BMJ. (2014) 348:g2272. doi: 10.1136/bmj.g2272 
12. Arab L. Biomarkers of fat and fatty acid intake. J Nutr. (2003) 133(Suppl. 3):925S-32S. doi: 10.1093/jn/133.3.925S

13. Patterson E, Wall R, Fitzgerald GF, Ross RP, Stanton C. Health implications of high dietary omega- 6 polyunsaturated fatty acids. J Nutr Metab. (2012) 2012:539426. doi: 10.1155/2012/ 539426

14. Forouhi NG, Imamura F, Sharp SJ, Koulman A, Schulze MB, Zheng J, et al. Association of plasma phospholipid n-3 and n-6 polyunsaturated fatty acids with type 2 diabetes: the EPIC-InterAct case-cohort study. PLoS Med. (2016) 13:e1002094. doi: 10.1371/journal.pmed. 1002094

15. Yary T, Voutilainen S, Tuomainen TP, Ruusunen A, Nurmi T, Virtanen JK. Serum n-6 polyunsaturated fatty acids, Delta5- and Delta6-desaturase activities, and risk of incident type 2 diabetes in men: the kuopio ischaemic heart disease risk factor study. Am J Clin Nutr. (2016) 103:133743. doi: 10.3945/ajen.115.128629

16. Wu JHY, Marklund M, Imamura F, Tintle N, Ardisson Korat AV, de Goede J, et al. Omega- 6 fatty acid biomarkers and incident type 2 diabetes: pooled analysis of individual-level data for 39740 adults from 20 prospective cohort studies. Lancet Diabetes Endocrinol. (2017) 5:965-74. doi: 10.1016/S2213-8587(17) 30307-8

17. Steffen BT, Steffen LM, Tracy R, Siscovick D, Jacobs D, Liu K, et al. Ethnicity, plasma phospholipid fatty acid composition and inflammatory/endothelial activation biomarkers in the multi-ethnic study of atherosclerosis (MESA). Eur J Clin Nutr. (2012) 66:600-5. doi: 10.1038/ejcn. 2011.215

18. Takkunen MJ, Schwab US, de Mello VD, Eriksson JG, Lindstrom J, Tuomilehto J, et al. Longitudinal associations of serum fatty acid composition with type 2 diabetes risk and markers of insulin secretion and sensitivity in the finnish diabetes prevention study. Eur J Nutr. (2016) 55:96779. doi: 10.1007/s00394-015-0911-4

19. Folch J, Lees M, Sloane Stanley GH. A simple method for the isolation and purification of total lipides from animal tissues. J Biol Chem. (1957) 226:497-509. doi: 10.1016/S0021-9258(18) 64849-5

20. American Diabetes A. 6. Glycemic targets. Diabetes Care. (2017) 40(Suppl. 1):S48-56. doi: $10.2337 / \mathrm{dc} 17-\mathrm{S} 009$

21. Flack JM, Adekola B. Blood pressure and the new ACC/AHA hypertension guidelines. Trends Cardiovasc Med. (2020) 30:1604. doi: $10.1016 /$ j.tcm.2019.05.003

22. Erkelens DW. Strategy for diagnosis and treatment of hyperlipidemia. Ther Umsch. (1990) 47:443-7.

23. R Core Team. R: A Language and Environment for Statistical Computing. Vienna: R Foundation for Statistical Computing (2020).

24. Akter S, Kurotani K, Sato M, Hayashi T, Kuwahara K, Matsushita Y, et al. High serum phospholipid dihomo-gamma-linoleic acid concentration and low delta5-desaturase activity are associated with increased risk of type 2 diabetes among japanese adults in the hitachi health study. J Nutr. (2017) 147:1558-66. doi: 10.3945/jn.117.248997

25. Burns JL, Nakamura MT, Ma DWL. Differentiating the biological effects of linoleic acid from arachidonic acid in health and disease. Prostagl Leukot Essent Fatty Acids. (2018) 135:1-4. doi: 10.1016/j.plefa.2018.05.004

26. Harris WS, Shearer GC. Omega-6 fatty acids and cardiovascular disease: friend, not foe? Circulation. (2014) 130:15624. doi: 10.1161/CIRCULATIONAHA.114.012534

27. Chowdhury R, Warnakula S, Kunutsor S, Crowe F, Ward HA, Johnson L, et al. Association of dietary, circulating, and supplement fatty acids with coronary risk: a systematic review and meta-analysis. Ann Intern Med. (2014) 160:398-406. doi: 10.7326/M13-1788

28. Suresh Y, Das UN. Protective action of arachidonic acid against alloxaninduced cytotoxicity and diabetes mellitus. Prostagl Leukot Essent Fatty Acids. (2001) 64:37-52. doi: 10.1054/plef.2000.0236

29. Krishna Mohan I, Das UN. Prevention of chemically induced diabetes mellitus in experimental animals by polyunsaturated fatty acids. Nutrition. (2001) 17:126-51. doi: 10.1016/S0899-9007(00)00468-8

30. Abbott KA, Burrows TL, Thota RN, Alex A, Acharya S, Attia J, et al. Association between plasma phospholipid omega-3 polyunsaturated fatty acids and type 2 diabetes is sex dependent: the hunter community study. Clin Nutr. (2019) 39:1059-66. doi: 10.1016/j.clnu.2019.04.007

31. Abbott KA, Veysey M, Lucock M, Niblett S, King K, Burrows T, et al. Sex-dependent association between erythrocyte n-3 PUFA and type 2 diabetes in older overweight people. Br J Nutr. (2016) 115:137986. doi: 10.1017/S0007114516000258

32. Abbott KA, Burrows TL, Thota RN, Acharya S, Garg ML. Do omega-3 PUFAs affect insulin resistance in a sex-specific manner? A systematic review and meta-analysis of randomized controlled trials. Am J Clin Nutr. (2016) 104:1470-84. doi: 10.3945/ajcn.116.138172

33. Decsi T, Kennedy K. Sex-specific differences in essential fatty acid metabolism. Am J Clin Nutr. (2011) 94(Suppl. 6):1914S-9S. doi: 10.3945/ajcn.110.000893

34. Nasreddine L, Chamieh MC, Ayoub J, Hwalla N, Sibai AM, Naja F. Sex disparities in dietary intake across the lifespan: the case of Lebanon. Nutr J. (2020) 19:24. doi: 10.1186/s12937-020-00543-x

35. Anderson WD, Soh JY, Innis SE, Dimanche A, Ma L, Langefeld CD, et al. Sex differences in human adipose tissue gene expression and genetic regulation involve adipogenesis. Genome Res. (2020) 30:137992. doi: $10.1101 /$ gr.264614.120

36. Tramunt B, Smati S, Grandgeorge N, Lenfant F, Arnal JF, Montagner A, et al. Sex differences in metabolic regulation and diabetes susceptibility. Diabetologia. (2020) 63:453-61. doi: 10.1007/s00125-019-05040-3

37. Weir NL, Nomura SO, Steffen BT, Guan W, Karger AB, Klein R, et al. Associations between omega- 6 polyunsaturated fatty acids, hyperinsulinemia and incident diabetes by race/ethnicity: the multi-ethnic study of atherosclerosis. Clin Nutr. (2020) 39:3031-41. doi: 10.1016/j.clnu.2020.01.003

38. Lankinen MA, Stancakova A, Uusitupa M, Agren J, Pihlajamaki J, Kuusisto J, et al. Plasma fatty acids as predictors of glycaemia and type 2 diabetes. Diabetologia. (2015) 58:2533-44. doi: 10.1007/s00125-015-3730-5

39. Zong G, Liu G, Willett WC, Wanders AJ, Alssema M, Zock PL, et al. Associations between linoleic acid intake and incident type 2 diabetes among U.S. Men and Women. Diabetes Care. (2019) 42:140613. doi: $10.2337 / \mathrm{dc} 19-0412$

40. Hodge AM, English DR, O’Dea K, Sinclair AJ, Makrides M, Gibson RA, et al. Plasma phospholipid and dietary fatty acids as predictors of type 2 diabetes: interpreting the role of linoleic acid. Am J Clin Nutr. (2007) 86:189-97. doi: 10.1093/ajcn/86.1.189

41. Onakpoya IJ, Posadzki PP, Watson LK, Davies LA, Ernst E. The efficacy of long-term conjugated linoleic acid (CLA) supplementation on body composition in overweight and obese individuals: a systematic review and meta-analysis of randomized clinical trials. Eur J Nutr. (2012) 51:12734. doi: 10.1007/s00394-011-0253-9

42. Paulweber B, Valensi P, Lindstrom J, Lalic NM, Greaves CJ, McKee M, et al. A European evidence-based guideline for the prevention of type 2 diabetes. Horm Metab Res. (2010) 42(Suppl. 1):S3-36. doi: 10.1055/s-00291240928

43. Dyson PA, Twenefour D, Breen C, Duncan A, Elvin E, Goff L, et al. Diabetes UK evidence-based nutrition guidelines for the prevention and management of diabetes. Diabet Med. (2018) 35:541-7. doi: 10.1111/ dme. 13603

44. Song Y, Manson JE, Buring JE, Liu S. A prospective study of red meat consumption and type 2 diabetes in middle-aged and elderly women: the women's health study. Diabetes Care. (2004) 27:210815. doi: $10.2337 /$ diacare.27.9.2108

45. Brostow DP, Odegaard AO, Koh WP, Duval S, Gross MD, Yuan JM, et al. Omega- 3 fatty acids and incident type 2 diabetes: the Singapore Chinese health study. Am J Clin Nutr. (2011) 94:520-6. doi: 10.3945/ajcn.110.009357

46. Alhazmi A, Stojanovski E, McEvoy M, Garg ML. Macronutrient intake and type 2 diabetes risk in middle-aged Australian women. Results from the Australian longitudinal study on women's health. Public Health Nutr. (2014) 17:1587-94. doi: 10.1017/S1368980013001870

47. Ericson U, Hellstrand S, Brunkwall L, Schulz CA, Sonestedt E, Wallstrom $\mathrm{P}$, et al. Food sources of fat may clarify the inconsistent role of dietary fat intake for incidence of type 2 diabetes. Am J Clin Nutr. (2015) 101:106580. doi: 10.3945/ajcn.114.103010

48. Dow C, Mangin M, Balkau B, Affret A, Boutron-Ruault MC, Clavel-Chapelon F, et al. Fatty acid consumption and incident type 2 diabetes: an 18-year 
follow-up in the female E3N (Etude Epidemiologique aupres des femmes de la Mutuelle Generale de l'Education Nationale) prospective cohort study. $\mathrm{Br} \mathrm{J}$ Nutr. (2016) 116:1807-15. doi: 10.1017/S0007114516003883

49. Neuenschwander $M$, Barbaresko J, Pischke CR, Iser $N$, Beckhaus J, Schwingshackl L, et al. Intake of dietary fats and fatty acids and the incidence of type 2 diabetes: a systematic review and dose-response meta-analysis of prospective observational studies. PLoS Med. (2020) 17:e1003347. doi: 10.1371/journal.pmed. 1003347

Conflict of Interest: The authors declare that the research was conducted in the absence of any commercial or financial relationships that could be construed as a potential conflict of interest.
Publisher's Note: All claims expressed in this article are solely those of the authors and do not necessarily represent those of their affiliated organizations, or those of the publisher, the editors and the reviewers. Any product that may be evaluated in this article, or claim that may be made by its manufacturer, is not guaranteed or endorsed by the publisher.

Copyright (c) $2021 \mathrm{Li}$, Shen, $\mathrm{Li}, \mathrm{Bi}, \mathrm{Bi}$, Che, Tian and Liu. This is an open-access article distributed under the terms of the Creative Commons Attribution License (CC $B Y)$. The use, distribution or reproduction in other forums is permitted, provided the original author(s) and the copyright owner(s) are credited and that the original publication in this journal is cited, in accordance with accepted academic practice. No use, distribution or reproduction is permitted which does not comply with these terms. 